\title{
Esophagectomy from then to now
}

\author{
Caitlin Takahashi ${ }^{1}$, Ravi Shridhar ${ }^{2}$, Jamie Huston $^{3}$, Kenneth Meredith ${ }^{3,4}$ \\ ${ }^{1}$ Department of Surgery, Naval Medical Center Portsmouth, Portsmouth, VA, USA; ${ }^{2}$ Department of Radiation Oncology, Florida Hospital Cancer \\ Institute, Orlando, FL, USA; ${ }^{3}$ Department of Gastrointestinal Oncology, Sarasota Memorial Healthcare System, Sarasota, FL, USA; ${ }^{4}$ Department of \\ Gastrointestinal Oncology, Florida State University College of Medicine, Tallahassee, FL, USA \\ Contributions: (I) Conception and design: All authors; (II) Administrative support: None; (III) Provision of study materials or patients: None; (IV) \\ Collection and assembly of data: J Huston; (V) Data analysis and interpretation: C Takahashi, R Shridhar, K Meredith; (VI) Manuscript writing: All \\ authors; (VII) Final approval of manuscript: All authors. \\ Correspondence to: Kenneth Meredith, MD, FACS. Professor, Florida State University College of Medicine; Director, Gastrointestinal Oncology, \\ Sarasota Memorial Institute for Cancer Care, Florida State University College of Medicine, 1950 Arlington Suite 101, Sarasota, FL, USA. \\ Email: Kenneth-Meredith@smh.com; kensurg@hotmail.com.
}

\begin{abstract}
We have come a long way from the onset of surgery for esophageal cancer. Surgical resection is pivotal for the long-term survival in patients with locally advanced esophageal cancer. Moreover, advancements in post-operative care and surgical techniques have contributed to reductions in morbidity. More recently minimally invasive esophagectomy has been increasingly used in patients undergoing esophageal cancer resection. Potential advantages of MIE include: the decreased pulmonary complications, lower post-operative wound infection, decreased post-operative pain, and decreased length of hospitalization. The application of robotics to esophageal surgery is becoming more widespread. Robotic esophageal surgery has potential advantages over the known limitations of laparoscopic and thoracoscopic approaches to esophagectomy while adhering to the benefits of the minimally invasive approach. This paper is a review of the evolution from open esophagectomy to the most recent robotic approach.
\end{abstract}

Keywords: Transhiatal esophagectomy; transthoracic esophagectomy; robotic esophagectomy

Submitted Aug 01, 2018. Accepted for publication Aug 17, 2018.

doi: 10.21037/jgo.2018.08.15

View this article at: http://dx.doi.org/10.21037/jgo.2018.08.15

\section{Introduction}

Esophageal cancer is increasing in incidence worldwide. In 2018 , it is estimated there will be 17,290 new cases of esophageal cancer with 15,850 dying from the disease in the USA (1). The overall survival for patients with esophageal cancer remains poor despite improvements in multidisciplinary care. The current approach to locally advanced esophageal cancer includes neoadjuvant chemoradiation followed by surgical resection (2). This multi-modality approach has demonstrated an improvement in survival for patients with advanced non-metastatic disease $(3,4)$.

Esophageal resection is crucial for improving the overall survival in patients with esophageal cancer. While survival for patients with resected esophageal cancer has improved with the institution of multidisciplinary treatment, the morbidity from esophagectomy remains high at 30-60\% (5-12). Traditionally, esophagectomy is performed via 2-3 large incisions via trans-abdominal [transhiatal (TH)], transthoracic [Ivor Lewis (ILE)] or three-field (McKeown approach) (13-18). The transhiatal approach is performed with an abdominal and left neck incision and esophageal to gastric anastomosis is performed in the left neck. Whereas ILE is performed using an abdominal incision and a right posterolateral thoracotomy incision with anastomosis performed within the right chest. The McKeown approach begins in the right chest, then progresses to the abdomen with anastomosis performed in the left neck.

The utilization of minimally invasive techniques for esophageal resection (MIE) offers some potential advantages over the conventional open esophagectomy. Some authors 
have demonstrated faster recovery times, decrease in blood loss, decrease in post-operative morbidities and shorter length or hospitalization, with comparable oncologic outcomes (19). Retrospective reviews have demonstrated MIE does not compromise oncologic principles and is safe compared to traditional open esophagectomy for esophageal cancer (20-24). Moreover, the Ivor Lewis approach when performed via a minimally invasive approach has the potential to substantially reduce pulmonary complications, a significant morbidity associated with the open approach.

The evolution of surgery to include robotics has expanded the capabilities of performing complex operations via minimally invasive techniques. The application of robotic technology to esophagectomy is proving to have some advantages over conventional minimally invasive techniques such as an increase accuracy in dissection through improved visualization via 3-dimensional camera and improved maneuverability through articulation of instruments (25). The utilization of thoracoscopic, laparoscopic, and robotic approaches to esophagectomy have shown to result in equivalent oncologic outcomes compared to open techniques (26-29) with some authors demonstrating reductions in post-operative morbidity (30,31).

\section{Methods}

This study was approved by the institutional review board at Sarasota Memorial Hospital and deemed as exempt (IRB\#15-onc-23). A query was performed utilizing PubMed with keywords "open esophagectomy, minimally invasive esophagectomy, robotic esophagectomy, Ivor Lewis esophagectomy, trans-hiatal esophagectomy and trans-thoracic esophagectomy" to identify existing peer reviewed manuscripts reporting outcomes associated with open, minimally invasive and robotic esophagectomy. Onehundred and fifty-seven manuscripts were identified and fifty-one were included in this review.

\section{Transthoracic vs. transhiatal approach}

In the 1990s, research was conflicted on whether the transhiatal esophagectomy or the transthoracic (Ivor Lewis) esophagectomy was superior. Bolton et al. investigated 55 patients between 1981 to 1990 who underwent transthoracic or trans-hiatal esophagectomy. They found the transhiatal approach had significantly lower operative mortality, lower cardiopulmonary complications and a shorter ICU stay (32). However, Jauch et al. in their series of 49 patients between 1982 to 1989 found no significant difference in mortality or overall survival rates between trans-hiatal vs. trans-thoracic esophagectomy but they did demonstrate an increase in anastomotic leak in the transhiatal group (33). Several additional studies published between 1993 and 1996 corroborated these findings that trans-hiatal and trans-thoracic approach are very similar in terms of postoperative complications, hospital mortality, and overall survival rates $(34,35)$.

More recently it has been suggested that transhiatal approaches have a statistically significant increase in rates of anastomotic leak. Homesh et al. in 84 patients, 43 receiving a transhiatal esophagectomy and 41 receiving a transthoracic esophagectomy for esophageal cancer; They reported no significant difference in ICU stay, blood transfusions and mean hospital stay. However the transhiatal approach had a much higher rate of anastomotic leak compared to transthoracic esophagectomy; $21 \%$ vs. $12 \%$, $\mathrm{P}=0.001$ (36). Klink et al. specifically investigated patients with cancer of the middle and lower third of the esophagus with transhiatal versus transthoracic esophagectomy. They also discovered lower rate of anastomotic leak in the transthoracic group, along with decreased incidence of wound infections, recurrent laryngeal nerve injury and hospital stay (37). In addition, patients with T3 N1 tumors, Kutup et al. demonstrated that TTE achieved a higher rate of R0 resections, a higher lymph node yield, and resulted in a prolonged survival than THE (38). The best approach to esophagectomy continues to be up for debate.

\section{Open versus MIE}

A propensity score matched analysis performed on 1,727 patients who underwent open or minimally invasive esophagectomy for esophageal cancer demonstrated an increase in surgical re-intervention and anastomotic leaks in the MIE group (39). This study however had significantly higher complications in both groups compared to historical data $(19,20,35,40)$. Most studies since then have found no significant difference or decrease in post-operative outcomes with the minimally invasive approaches. The E2202 study showed feasibility and safety of performing MIE, with 30-day mortality of $2.1 \%$, anastomotic leaks $8.6 \%$, and 3-year overall survival of $58.4 \%$ (19). Maas et al. also demonstrated feasibility with no difference between open verses MIE with regards to post-operative morbidity, oncological outcome or 5-year survival. The MIE group did 
have a statistically significant shorter hospital stay than the open esophagectomy group (41).

Bierre et al. conducted one of the first randomized trials of MIE versus open esophagectomy (42). In the TIME trial, 59 patients were randomized to the MIE group and 56 patients were randomized to the open esophagectomy group. Open esophagectomy demonstrated higher pulmonary infection rates; $29 \%$ vs. 9\%; [relative risk (RR) 0.30, 95\% CI: 0.12-0.76; P=0.005]. Length of hospital stay was also lower in MIE patients (11 vs. 14 days; $\mathrm{P}=0.044$ ). With regards to in-hospital mortality, two patients in the minimally invasive group died from aspiration and mediastinitis after anastomotic leakage and a single patient in the open esophagectomy group died from anastomotic leakage. In a 3-year follow up of Biere's TIME trial participants' quality of life, MIE demonstrated superiority for global health, physical component, and pain (43). A small randomized controlled trial by Schoppmann et al. of 62 patients corroborated these results by demonstrating improved post-operative morbidity, transfusion rates and pulmonary complications in the MIE group versus the open cohort (44). Guo et al. has also confirmed decrease length of hospital stay and fewer pulmonary infections in MIE (45).

In 2016, Sihag et al. queried the Society of Thoracic Surgeons National Database to determine outcomes of MIE versus open esophagectomy (46). The analysis compared both open transthoracic $(n=1,291)$ and transhiatal $(n=214)$ against minimally invasive Ivor Lewis esophagectomy $(n=600)$ patients. Morbidity and mortality were similar between open and MIE. However, MIE was associated with longer median operative times (443.0 vs. 312.0 minutes; $\mathrm{P}<0.001)$, shorter length of hospitalization (9.0 vs. 10.0 days; $\mathrm{P}<0.001)$. Patients who underwent MIE had increased rates of empyema $(4.1 \%$ vs. $1.8 \% ; \mathrm{P}<0.001)$ and re-operation ( $9.9 \%$ vs. $4.4 \% ; \mathrm{P}<0.001$ ). Patients undergoing open esophagectomy had increased rates of post-operative transfusions (18.7\% vs. $14.1 \% ; \mathrm{P}=0.002)$, ileus $(4.5 \%$ vs. $2.2 \% ; \mathrm{P}=0.002)$ and wound infections $(6.3 \%$ vs. $2.3 \%$; $\mathrm{P}<0.001)$. Propensity score-matched analysis corroborated these findings. Yerokun et al. confirmed these results in an analysis of the National Cancer Database (47). They identified 4,266 patients and 1,308 (30.6\%) underwent minimally invasive esophagectomy. Minimally invasive approaches were more often performed at academic centers or cancer centers. Additionally, MIE patients had shorter hospital stays (10 vs. 11 days; $\mathrm{P}=0.046)$ and more lymph nodes retrieved (15 vs. 13; $\mathrm{P}=0.016)$. There were equivalent R0 resections, 30-day mortality, and readmissions. There were no differences in survival between the matched groups at 3 years.

A meta-analysis of 48 studies by Zhou et al. comparing 14,311 patients undergoing MIE and open esophagectomy demonstrated that compared to open patients, MIE patients experienced less pulmonary complications, pulmonary embolism, in-hospital mortality, and arrhythmias, with no differences in leakage from the anastomosis (48). The evidence has resulted in a push toward using a minimally invasive approach as the standard of care for esophagectomy.

\section{Hybrid approach}

There have been few studies on a hybrid approach to esophagectomy. Woodard et al. investigated the feasibility and safety of hybrid esophagectomy in 131 patients, demonstrating 30- and 90-day mortality rates of $0.8 \%$ and $2.3 \%$ respectively, and overall survival at 1,3 and 5 years of $85.9 \%, 65.3 \%$ and $53.9 \%$ (49). Briez et al. further investigated the implication of a hybrid approach to esophagectomy on pulmonary complications. They discovered that post-operative pulmonary complications were much less in hybrid approach than open, $15.7 \%$ vs. $42.9 \%, \mathrm{P}<0.01$ (50). The hybrid approach is feasible with better outcomes than open esophagectomy but has not proven to be superior to MIE in hospital mortality or morbidity.

\section{Esophagectomy volume and outcome}

While the type of operation performed can dictate patient outcomes, equally as important is the experience and number of esophagectomies performed by the surgeon. The previously discussed TIME trial by Bierre et al., reported that patients who underwent esophagectomy had an improvement in morbidity and mortality if they had operations performed by high volume surgeons regardless of open or minimally invasive technique. Derogar et al. in a population-based cohort study of 1,335 patients with esophageal cancer undergoing esophagectomy from 1987 to 2005 confirmed these findings. They found a $23 \%$ reduction in mortality when esophagectomy was performed by high volume surgeons which was independent of hospital volume (51).

\section{Robotic approaches to esophagectomy}

The application of robotics in surgery has shown the 
potential to increase accuracy in dissection through improved maneuverability and visualization while minimizing post-operative recovery time and blood loss. Robotic assisted Ivor Lewis esophagectomy is a technique with some potential advantages over conventional laparoscopic and thoracoscopic approach to esophagectomy. Robotic arms have the ability to articulate more than even a surgeon's capability. Resultantly, these movements closely mirror open surgical techniques. It is these potential benefits that has led to a significant increase in the utilization of robotics for esophageal resection over the last 5 years. Although there is the implication of significant increase in cost of utilizing the robotic for esophageal resection, these costs may be offset from decreased blood loss, morbidity, and length of hospitalization (52).

\section{Outcomes with robotic approaches to esophagectomy}

Robotic approach to esophagectomy has proven safe and feasible since its beginning. Dunn et al. investigated outcomes of 40 patients who underwent transhiatal robotic esophagectomy demonstrating practicality of robotic esophagectomy. Median operative time and blood loss were 311 minutes and $97 \mathrm{~mL}$ and the median length of hospital stay was 9 days. R0 resection achieved was $94.7 \%$ and 30 -day mortality was $2.5 \%$ (25). Moreover, Puntambekar displayed feasibility in utilizing robotics for the transthoracic approach, with mean operative time of 204.94 minutes, mean hospital stay of 10 days, and $19.28 \%$ of postoperative complications (52). In a series of 147 robotic assisted Ivor Lewis esophagectomies (RAIL) by Meredith et al. RAIL had similar operative times to esophagectomies performed via other minimally invasive approaches; median time of 346 minutes versus median time of 320 minutes respectively $(53,54)$. Meredith et al. also achieved $100 \% \mathrm{R} 0$ resection with median nodal harvest of 20.4. The median length of stay in the ICU and hospital stay were 2 days and 9 days respectively and the complication rate was $25.2 \%$ (53).

Coker et al. reported that RAIL had overall fewer complications (cardiovascular, wound, pulmonary, and overall) compared to open Ivor Lewis esophagectomies (55). Additionally, length of hospitalization was significantly lower in the RAIL versus open. However, in a series by Mori et al. comparing robotically-assisted transhiatal esophagectomy (RATE), a transthoracic approach resulted in increased pulmonary complications (56). Unfortunately, there were only 22 patients in the robotic transhiatal group and these were compared to conventional transthoracic not robotic esophagectomies. Additionally, aspiration events, which are known to be higher in the $\mathrm{TH}$ approach, were not reported in this series.

As the length of operation for RAIL continues to decrease, the rates of pulmonary complications also are reduced as demonstrated by Hernandez et al. who reported decreasing rates of pneumonias as operative times decreased (57). Conversely, robotic assisted transhiatal esophagectomy has increased rates of major complications compared to robotic Ivor Lewis approach. These include increased wound complications, a higher incidence of recurrent laryngeal nerve injuries, aspiration, and anastomotic leaks $(25,56,58,59)$. Other authors have confirmed anastomotic leaks, pneumonias, wound complications, and any complication were all decreased in the cohort of patients who had robotic Ivor Lewis esophagectomy (56).

While the R0 resection rates for RATE and RAIL may be comparable, the ability to perform an extended lymphadenectomy of the mediastinal lymph nodes which may have staging and survival implications are benefited by patients undergoing RAIL $(31,52,55,58)$. Lymph node dissection in RATE is limited by the robot's ability to fit through the diaphragmatic hiatus and resultant limited view into the thoracic cavity from the abdomen. Therefore, the superior extent of lymph node dissection and subsequent lymph node harvest in RATE is inferior compared to RAIL. In comparison of our own data with minimally invasive esophagectomy, RAIL was superior to other techniques with median lymph node retrieval for open IL, RAIL, MIE IL, and MIE transhiatal being 10, 20, 14, and 9, $\mathrm{P}=0.001$ (40).

\section{Conclusions}

There have been significant improvements made in the surgical resection of esophageal cancer from the highly morbid open esophagectomy. The advancements in MIE have improved post-operative outcomes significantly to result in shortened length of hospitalization, fewer pulmonary complications, and improved quality of life. Most importantly, there is no compromise in oncologic outcomes such as nodal harvests, margin status, and survival compared to open approaches. There is still some debate on the benefits of transthoracic versus transhiatal approaches, with most recent evidence demonstrating transthoracic to 
be superior. With the added benefits of robotic surgery, esophagectomies have evolved even further. The robotic assisted Ivor Lewis esophagectomy demonstrates similar outcomes compared to other MIE techniques. While there is a significant learning curve associated with robotic assisted Ivor Lewis esophagectomy, as the number of cases performed increases, the post-operative complications and length of operation and is decreased. More data is needed to compare robotic assisted techniques to existing minimally invasive techniques in order to determine the best approach for esophageal resection.

\section{Acknowledgements}

None.

\section{Footnote}

Conflicts of Interest: The authors have no conflicts of interest to declare.

\section{References}

1. Siegel RL, Miller KD, Jemal A. Cancer statistics, 2016. CA Cancer J Clin 2016;66:7-30.

2. NCCN Clinical Practice Guidelines in Oncology: Esophageal and Esophagogastric Junction Cancers. 2013. Available online: http://www.isesnet.org/wp-content/ uploads/2013/02/NCCN-esophagus-2012.pdf

3. Sjoquist KM, Burmeister BH, Smithers BM, et al. Survival after neoadjuvant chemotherapy or chemoradiotherapy for resectable oesophageal carcinoma: an updated metaanalysis. Lancet Oncol 2011;12:681-92.

4. van Hagen P, Hulshof MC, van Lanschot JJ, et al. Preoperative chemoradiotherapy for esophageal or junctional cancer. N Engl J Med 2012;366:2074-84.

5. Atkins BZ, Shah A, Hutcheson K, et al. Reducing hospital morbidity and mortality following esophagectomy. Ann Thorac Surg 2004;78:1170-6.

6. Bedenne L, Michel P, Bouche O, et al. Chemoradiation followed by surgery compared with chemoradiation alone in squamous cancer of the esophagus: FFCD 9102. J Clin Oncol 2007;25:1160-8.

7. Chang AC, Ji H, Birkmeyer NJ, et al. Outcomes after transhiatal and transthoracic esophagectomy for cancer. Ann Thorac Surg 2008;85:424-9.

8. Gebski V, Burmeister B, Smithers BM, et al. Survival benefits from neoadjuvant chemoradiotherapy or chemotherapy in oesophageal carcinoma: a meta-analysis. Lancet Oncol 2007;8:226-34.

9. Jin J, Liao Z, Zhang Z, et al. Induction chemotherapy improved outcomes of patients with resectable esophageal cancer who received chemoradiotherapy followed by surgery. Int J Radiat Oncol Biol Phys 2004;60:427-36.

10. Karl RC, Schreiber R, Boulware D, et al. Factors affecting morbidity, mortality, and survival in patients undergoing Ivor Lewis esophagogastrectomy. Ann Surg 2000;231:635-43.

11. Kelsen DP, Winter KA, Gunderson LL, et al. Longterm results of RTOG trial 8911 (USA Intergroup 113): a random assignment trial comparison of chemotherapy followed by surgery compared with surgery alone for esophageal cancer. J Clin Oncol 2007;25:3719-25.

12. Pennathur A, Luketich JD, Landreneau RJ, et al. Long-term results of a phase II trial of neoadjuvant chemotherapy followed by esophagectomy for locally advanced esophageal neoplasm. Ann Thorac Surg 2008;85:1930-6; discussion 1936-7.

13. de Boer AG, van Lanschot JJ, van Sandick JW, et al. Quality of life after transhiatal compared with extended transthoracic resection for adenocarcinoma of the esophagus. J Clin Oncol 2004;22:4202-8.

14. Earlam R, Cunha-Melo JR. Oesophageal squamous cell carcinoma: I. A critical review of surgery. Br J Surg 1980;67:381-90.

15. Hulscher JB, van Sandick JW, de Boer AG, et al. Extended transthoracic resection compared with limited transhiatal resection for adenocarcinoma of the esophagus. N Engl J Med 2002;347:1662-9.

16. Orringer MB, Marshall B, Chang AC, et al. Two thousand transhiatal esophagectomies: changing trends, lessons learned. Ann Surg 2007;246:363-72; discussion 372-64.

17. Rizzetto C, DeMeester SR, Hagen JA, et al. En bloc esophagectomy reduces local recurrence and improves survival compared with transhiatal resection after neoadjuvant therapy for esophageal adenocarcinoma. J Thorac Cardiovasc Surg 2008;135:1228-36.

18. Wu PC, Posner MC, Wu PC, et al. The role of surgery in the management of oesophageal cancer. Lancet Oncol 2003;4:481-8.

19. Luketich JD, Pennathur A, Awais O, et al. Outcomes after minimally invasive esophagectomy: review of over 1000 patients. Ann Surg 2012;256:95-103.

20. Santillan AA, Farma JM, Meredith KL, et al. Minimally invasive surgery for esophageal cancer. J Natl Compr Canc Netw 2008;6:879-84. 
21. Verhage RJ, Hazebroek E, Boone J, et al. Minimally invasive surgery for esophageal cancer. Minerva Chir 2009;64:135-46.

22. Safranek PM, Cubitt J, Booth M, et al. Review of open and minimal access approaches to oesophagectomy for cancer. Br J Surg 2010; 97:1845-53.

23. Singh RK, Pham T, Diggs B, et al. Minimally invasive esophagectomy provides equivalent oncologic outcomes to open esophagectomy for locally advanced (stage II or III) esophageal carcinoma. Arch Surg 2011;146:711-4.

24. Straatman J, van der Wielen N, Cuesta M, et al. Minimally Invasive Versus Open Esophageal Resection: Three-year Follow-up of the Previously Reported Randomized Controlled Trial: the TIME Trial. Ann Surg 2017;266:232-6.

25. Dunn DH, Johnson EM, Morphew JA, et al. Robotassisted transhiatal esophagectomy: a 3-year single-center experience. Dis Esophagus 2013;26:159-66.

26. Bizekis C, Kent MS, Luketich JD, et al. Initial experience with minimally invasive Ivor Lewis esophagectomy. Ann Thorac Surg 2006;82:402-6; discussion 406-7.

27. Law SY, Fok M, Wei WI, et al. Thoracoscopic esophageal mobilization for pharyngolaryngoesophagectomy. Ann Thorac Surg 2000;70:418-22.

28. Luketich JD, Alvelo-Rivera M, Buenaventura PO, et al. Minimally invasive esophagectomy: outcomes in 222 patients. Ann Surg 2003;238:486-94; discussion 494-5.

29. Merritt RE. Initial experience of total thoracoscopic and laparoscopic Ivor Lewis esophagectomy. J Laparoendosc Adv Surg Tech A 2012;22:214-9.

30. Luketich JD, Pennathur A, Awais O, et al. Outcomes after minimally invasive esophagectomy: review of over 1000 patients. Ann Surg 2012;256:95-103.

31. de la Fuente SG, Weber J, Hoffe SE, et al. Initial experience from a large referral center with roboticassisted Ivor Lewis esophagogastrectomy for oncologic purposes. Surg Endosc 2013;27:3339-47.

32. Bolton JS, Sardi A, Bowen JC, et al. Transhiatal and transthoracic esophagectomy: a comparative study. J Surg Oncol 1992;51:249-53.

33. Jauch KW, Bacha EA, Denecke H, et al. Esophageal carcinoma: prognostic features and comparison between blunt transhiatal dissection and transthoracic resection. Eur J Surg Oncol 1992;18:553-62.

34. Goldminc M, Maddern G, Le Prise E, et al. Oesophagectomy by a transhiatal approach or thoracotomy: a prospective randomized trial. Br J Surg 1993;80:367-70.
35. Stark SP, Romberg MS, Pierce GE, et al. Transhiatal versus transthoracic esophagectomy for adenocarcinoma of the distal esophagus and cardia. Am J Surg 1996;172:47881; discussion 481-2.

36. Homesh NA, Alsabahi AA, Al-Agmar MH, et al. Transhiatal versus transthoracic resection for oesophageal carcinoma in Yemen. Singapore Med J 2006;47:54-9.

37. Klink CD, Binnebösel M, Otto J, et al. Intrathoracic versus cervical anastomosis after resection of esophageal cancer: a matched pair analysis of 72 patients in a single center study. World J Surg Oncol 2012;10:159.

38. Kutup A, Nentwich MF, Bollschweiler E, et al. What should be the gold standard for the surgical component in the treatment of locally advanced esophageal cancer: transthoracic versus transhiatal esophagectomy. Ann Surg 2014;260:1016-22.

39. Seesing MFJ, Gisbertz SS, Goense L, et al. A Propensity Score Matched Analysis of Open Versus Minimally Invasive Transthoracic Esophagectomy in the Netherlands. Ann Surg 2017;266:839-46.

40. Yamamoto M, Weber J, Karl R, et al. Minimally invasive surgery for esophageal cancer: Review of the literature and the institutional experience. Cancer Control 2013;20:130-7.

41. Maas KW, Biere SS, Scheepers JJ, et al. Laparoscopic versus open transhiatal esophagectomy for distal and junction cancer. Rev Esp Enferm Dig 2012;104:197-202.

42. Biere SS, Van Berge Henegouwen M, Maas K, et al. Minimally invasive versus open oesophagectomy for patients with oesophageal cancer: a multicentre, open-label, randomised controlled trial. Lancet 2012:379:1887-92.

43. Maas KW, Cuesta MA, van Berge Henegouwen MI, et al. Quality of Life and Late Complications After Minimally Invasive Compared to Open Esophagectomy: Results of a Randomized Trial. World J Surg 2015;39:1986-93.

44. Schoppmann SF, Prager G, Langer FB, et al. Open versus minimally invasive esophagectomy: a single-center case controlled study. Surg Endosc 2010;24:3044-53.

45. Guo M, Xie B, Sun X, et al. A comparative study of the therapeutic effect in two protocols: video-assisted thoracic surgery combined with laparoscopic versus right open transthoracic esophagectomy for esophageal cancer management. Chin Ger Clin Oncol 2013;12:1268-71.

46. Sihag S, Kosinski AS, Gaissert HA, et al. Minimally Invasive Versus Open Esophagectomy for Esophageal Cancer: A Comparison of Early Surgical Outcomes From The Society of Thoracic Surgeons National Database. Ann Thorac Surg 2016;101:1281-8; discussion 1288-9. 
47. Yerokun BA, Sun Z, Jeffrey Yang CF, et al. Minimally Invasive Versus Open Esophagectomy for Esophageal Cancer: A Population-Based Analysis. Ann Thorac Surg 2016;102:416-23.

48. Zhou C, Zhang L, Wang H, et al. Superiority of Minimally Invasive Oesophagectomy in Reducing In-Hospital Mortality of Patients with Resectable Oesophageal Cancer: A Meta-Analysis. PLoS One 2015;10:e0132889.

49. Woodard GA, Crockard JC, Clary-Macy C, et al. Hybrid minimally invasive Ivor Lewis esophagectomy after neoadjuvant chemoradiation yields excellent long-term survival outcomes with minimal morbidity. J Surg Oncol 2016;114:838-47.

50. Briez N, Piessen G, Torres F, et al. Effects of hybrid minimally invasive oesophagectomy on major postoperative pulmonary complications. Br J Surg 2012;99:1547-53.

51. Derogar M, Sadr-Azodi O, Johar A, et al. Hospital and surgeon volume in relation to survival after esophageal cancer surgery in a population-based study. J Clin Oncol 2013;31:551-7.

52. Puntambekar S, Kenawadekar R, Kumar S, et al. Robotic transthoracic esophagectomy. BMC Surg 2015;15:47.

53. Meredith K, Huston J, Andacoglu O, et al. Safety and

Cite this article as: Takahashi C, Shridhar R, Huston J, Meredith K. Esophagectomy from then to now. J Gastrointest Oncol 2018;9(5):903-909. doi: 10.21037/jgo.2018.08.15 feasibility of robotic-assisted Ivor-Lewis esophagectomy. Dis Esophagus 2018;31:doy005.

54. Yamamoto S, Kawahara K, Maekawa T, et al. Minimally invasive esophagectomy for stage I and II esophageal cancer. Ann Thorac Surg 2005;80:2070-5.

55. Boone J, Schipper ME, Moojen WA, et al. Robot-assisted thoracoscopic oesophagectomy for cancer. Br J Surg 2009;96:878-86.

56. Mori K, Yamagata Y, Aikou S, et al. Short-term outcomes of robotic radical esophagectomy for esophageal cancer by a nontransthoracic approach compared with conventional transthoracic surgery. Dis Esophagus 2016;29:429-34.

57. Hernandez JM, Dimou F, Weber J, et al. Defining the learning curve for robotic-assisted esophagogastrectomy. J Gastrointest Surg 2013;17:1346-51.

58. Cerfolio RJ, Bryant AS, Hawn MT. Technical aspects and early results of robotic esophagectomy with chest anastomosis. J Thorac Cardiovasc Surg 2013;145:90-6.

59. Coker AM, Barajas-Gamboa JS, Cheverie J, et al. Outcomes of robotic-assisted transhiatal esophagectomy for esophageal cancer after neoadjuvant chemoradiation. J Laparoendosc Adv Surg Tech A 2014;24:89-94. 